Bull. Austral. Math. Soc.

Vol. 53 (1996) [149-157]

\title{
GENERALISED DISCRIMINANTS
}

\section{C.F. Woodcock and P.R. Graves-Morris}

In this paper, we establish the generalised discriminant theorem governing uniqueness of symmetric polynomials in $n$ variables of least total degree $d_{n, k}$ which vanish when any $k+1$ of the variables are equal.

\section{INTRODUCTION}

The symmetric polynomial in the variables $x_{1}, x_{2}, \ldots, x_{n}$ of least total degree which vanishes when any two of these variables are equal is

$$
P_{n, 1}(\mathbf{x})=\prod_{1 \leqslant i<j \leqslant n}\left(x_{i}-x_{j}\right)^{2},
$$

where $\mathrm{x}:=\left(x_{1}, x_{2}, \ldots, x_{n}\right)$. This polynomial is called the discriminant polynomial [2]. It has total degree $n(n-1)$ and degree $2(n-1)$ in each of the $x_{i}$.

Equation (20) gives the explicit formula which generalises $P_{n, 1}(x)$ and is the (nontrivial) symmetric polynomial of least total degree in $x_{1}, x_{2}, \ldots, x_{n}$ which vanishes when any $k+1$ of the $x_{i}$ are equal. We call this polynomial the generalised discriminant and we show that it is unique up to an overall scale factor. These new results are summarised by Theorem 1 .

\section{GENERALISED Discriminants}

We shall show that for each $n, k \geqslant 1$ there is, up to a constant multiple, a unique (non-zero) symmetric polynomial $P_{n, k}\left(x_{1}, x_{2}, \ldots, x_{n}\right)$ of least total degree (with coefficients in a field of characteristic zero) which vanishes whenever $k+1$ of the $x_{i}$ are equal. We shall further show that

(i) $P_{n, k}\left(x_{1}, x_{2}, \ldots, x_{n}\right)$ is a homogeneous polynomial,

(ii) $\operatorname{deg}\left\{P_{n, k}\right\}=k q(q-1)+2 r q$, where

$$
q=[n / k] \text { and } r=n-q k,
$$

Received 20 April 1995.

P.R. Graves-Morris gratefully acknowledges an Erskine fellowship at Canterbury University. We are grateful to Dr. J.R. Merriman and Mr. R. Thukral for some helpful discussions and symbolic calculations.

Copyright Clearance Centre, Inc. Serial-fee code: $0004-9729 / 96 \$$ A2.00+0.00. 
and

(iii) $P_{n, k}\left(x_{1}, x_{2}, \ldots, x_{n}\right)$ has degree $2[(n-1) / k]$ in each variable $x_{i}$.

For brevity, and where appropriate, we let $\mathbf{x}:=\left(x_{1}, x_{2}, \ldots, x_{n}\right)$ denote the variables.

Example 1. Case $k=1$. In the introduction, we observed that

$$
P_{n, 1}(\mathrm{x})=\prod_{1 \leqslant i<j \leqslant n}\left(x_{i}-x_{j}\right)^{2}
$$

is called the discriminant polynomial in $n$ variables. It is the symmetric polynomial of least degree which vanishes when any two of $\left\{x_{i}\right\}$ are equal and it is totally symmetric with respect to interchange of $\left\{x_{i}\right\}$. It is unique up to a non-zero constant multiplier. We note that $P_{n, 1}(x)$ is of total degree $n(n-1)$ in accord with (ii) and it is of degree $2(n-1)$ in each $x_{i}$ individually, in accord with (iii) above.

Before going on to the next example, we show that if $P(\mathbf{x})$ is to be a generalised discriminant polynomial of degree $d$ say, then it is necessarily homogeneous. Consider the effect of a dilation $\mathbf{x} \rightarrow \alpha \mathbf{x}$ under which

$$
P(x) \rightarrow P(x ; \alpha) \equiv \sum_{j=0}^{d} p_{j}(x) \alpha^{j}
$$

$P(\mathbf{x} ; \alpha)$ is a polynomial in the variables $\mathbf{x}, \alpha$ and it vanishes whenever $k+1$ of its variables $x_{i}$ are equal; each $p_{j}(\mathbf{x})$ inherits this property. By construction, each $p_{j}(\mathbf{x})$ is of total degree $j$. But, by hypothesis, $d$ is minimal, and so $p_{j}(\mathbf{x}) \equiv 0$ for $j<d$. Consequently $P(\mathbf{x})$ and each $P_{n, k}(\mathbf{x})$ must be homogeneous polynomials.

ExAmPle 2. Case $n=3, k=2$.

$$
P_{3,2}(\mathbf{x})=\left(x_{1}-x_{2}\right)^{2}+\left(x_{2}-x_{3}\right)^{2}+\left(x_{3}-x_{1}\right)^{2}
$$

is a non-trivial symmetric polynomial in $x_{1}, x_{2}, x_{3}$ and it vanishes identically if $x_{1}=$ $x_{2}=x_{3}$. The only polynomial of lesser degree with these properties is $P(x)=0$. Because the required polynomial has to be homogeneous, it readily follows that $P_{3,2}(\mathrm{x})$ is unique up to an overall constant multiplier.

Construction of the generalised discriminant $P_{n, k}(\mathrm{x})$ : Let the index set $\{1,2, \ldots, n\}$ denote the vertices of a graph $G$, and let $G$ be the disjoint union of cliques $C_{1}, C_{2}, \ldots, C_{k}$, where $C_{1}, C_{2}, \ldots, C_{r}$ each have $q+1$ vertices and $C_{r+1}, C_{r+2}, \ldots, C_{k}$ each have $q$ vertices. (A clique with $q$ vertices is the complete subgraph formed with these vertices [6].) Let $E$ denote the set of all ordered pairs 
$(i, j)$ in which $i<j$ and $i, j$ belong to the same clique. Thus $E$ is the set of (directed) edges of $G$, and we form

$$
P_{E}(\mathrm{x}):=\prod_{\ell=1}^{k} \prod_{\substack{i<j \\ i, j \in C_{\ell}}}\left(x_{i}-x_{j}\right)^{2}=\prod_{(i, j) \in E}\left(x_{i}-x_{j}\right)^{2} .
$$

We construct the generalised discriminant $P_{n, k}(\mathbf{x})$ as the symmetrised form of $p_{E}(\mathbf{x})$,

$$
P_{n, k}(\mathbf{x}):=\sum_{\sigma \in S_{n}} \prod_{(i, j) \in E}\left(x_{\sigma(i)}-x_{\sigma(j)}\right)^{2}
$$

where $S_{n}$ is the group of all permutations of $\{1,2, \ldots, n\}$.

It is easily verified that $P_{n, k}(\mathrm{x})$ defined by (4) possesses the properties (i), (ii), (iii) above. Property (i) is obvious. Property (ii) follows by counting the contributions $q(q+1)$ to the degree from each clique $C_{1}, \ldots, C_{r}$ and $q(q-1)$ from each clique $C_{r+1}, \ldots, C_{k}$. We denote the degree of $P_{n, k}(\mathbf{x})$ by

$$
\lambda_{n, k}:=k q(q-1)+2 r q .
$$

Property (iii) follows by checking the power of $x_{1}$ and considering separately the two cases of $r=0$ and $r>0$.

It is also true that (4) expresses $P_{n, k}(\mathbf{x})$ as a sum of squares, each of which vanishes if any $k+1$ of the $x_{i}$ are equal. To see that $P_{n, k}(\mathbf{x})$ does not vanish identically when $k$ or fewer of the $x_{i}$ are equal, re-order the $x_{i}$ so that variables equal by value occur consecutively. Place the variables one by one in this order into the cliques $C_{1}, C_{2}, \ldots, C_{k}$ consecutively. In this way, the cliques have their prescribed numbers of elements, no clique contains two variables equal by value, and a non-zero term of the form (3) has been constructed.

Next we must prove that $P_{n, k}(\mathbf{x})$ is of minimal degree. To this end, we use the characterisation of $P_{n, k}(\mathbf{x})$ given in Lemma 1, and we need some preliminary definitions.

Definition: We define a lexicographical order $\geqslant$ on the set of monomials $x_{1}^{s_{1}} x_{2}^{s_{2}} \ldots x_{n}^{s_{n}}$ (each $s_{i} \geqslant 0$ ) by defining $x_{1}^{\ell_{1}} x_{2}^{\ell_{2}} \ldots x_{n}^{\ell_{n}} \geqslant x_{1}^{m_{1}} x_{2}^{m_{2}} \ldots x_{n}^{m_{n}}$ with equality if $\ell_{i}=m_{i} i=1,2, \ldots, n$ or inequality if $\ell_{i}>m_{i}$, where $i$ is the least index with $\ell_{i} \neq m_{i}$.

Definition: We define the leading monomial $\mathcal{M}(P)$ of a given polynomial $P(\mathbf{x})$ to be the greatest monomial (under the lexicographical order) with non-zero coefficient in $P(\mathbf{x})$.

Thus $\mathcal{M}(P)$ takes the form

$$
\mathcal{M}(P)=x_{1}^{\ell_{1}} x_{2}^{\ell_{2}} \ldots x_{n}^{\ell_{n}} \text { with } \ell_{1} \geqslant \ell_{2} \geqslant \ldots \geqslant \ell_{n}
$$


if $P(x)$ is symmetric in $x_{1}, x_{2}, \ldots, x_{n}$. The integers $\left(\ell_{1}, \ell_{2}, \ldots, \ell_{n}\right)$ provide a partial characterisation of $P(x)$, and $P(x)$ obviously has degree $\ell_{1}$ in the variable $x_{1}$ alone and, for that matter, degree $\ell_{1}$ in each $x_{i}$ if $P(x)$ is symmetric. We also have a hierarchical property: if

$$
P(\mathbf{x})=x_{1}^{\ell_{1}} Q\left(x_{2}, \ldots, x_{n}\right)+\text { lower order terms in } x_{1},
$$

then

$$
\mathcal{M}(P)=x_{1}^{\ell_{1}} \mathcal{M}(Q)
$$

Definition of THE ChaRACTER $H_{n, k}\left(\ell_{1}, \ell_{2}, \ldots, \ell_{n}\right)$ : If a polynomial $P(\mathbf{x})$ has the properties that

(a) $P(\mathbf{x})$ is homogeneous,

(b) $P(\mathrm{x})$ is symmetric in $x_{1}, x_{2}, \ldots, x_{n}$,

(c) $P(\mathbf{x})=0$ whenever $k+1$ of the $x_{i}$ are equal,

then $P(\mathbf{x})$ is said to have character $H_{n, k}\left(\ell_{1}, \ell_{2}, \ldots, \ell_{n}\right)$ where $\ell_{1}, \ell_{2}, \ldots, \ell_{n}$ are the powers in the leading monomial $x_{1}^{\ell_{1}} \ldots x_{n}^{\ell_{n}}$ of $P(\mathbf{x})$.

LEMMA 1. The generalised discriminant $P_{n, k}(\mathbf{x})$ defined by (4) has character $H_{n, k}\left(\ell_{1}, \ell_{2}, \ldots, \ell_{n}\right)$ where

$$
\ell_{i}:=\lambda_{n-i+1, k}-\lambda_{n-i, k}=2\left[\frac{n-i}{k}\right]
$$

and

$$
\ell_{1}+\ell_{2}+\ldots+\ell_{n}=\lambda_{n, k} .
$$

ProOF: The remarks associated with the Definition (5) were made to establish that $\ell_{1}=\lambda_{n, k}-\lambda_{n-1, k}=2[(n-1) / k]$. Equation (9) follows from (5) together with the hierarchical principle expressed by (7), and (10) reflects the decomposition (6).

In order to prove that $P_{n, k}(\mathbf{x})$ is of minimal degree, we consider an arbitrary polynomial $P(\mathrm{x})$ which satisfies condition (a), (b) and (c) above. Then $P(\mathrm{x})$ has total degree $\ell_{1}+\ell_{2}+\ldots+\ell_{n}$, it is of degree $\ell_{1}$ in each $x_{i}$ separately, and so $P(x)$ has character $H_{n, k}\left(\ell_{1}, \ldots, \ell_{n}\right)$. If we express

$$
P(\mathrm{x})=x_{1}^{\ell_{1}} Q\left(x_{2}, \ldots, x_{n}\right)+\text { lower order terms in } x_{1},
$$

then $Q(\mathbf{x})$ has character $H_{n-1, k}\left(\ell_{2}, \ldots, \ell_{n}\right)$ in the variables $x_{2}, \ldots, x_{n}$. This decomposition follows directly from the preceding definition of the character $H_{n, k}\left(\ell_{1}, \ldots, \ell_{n}\right)$ and from its hierarchical property.

Similarly, we may express

$$
P(\mathrm{x})=x_{1}^{\ell_{1}} x_{2}^{\ell_{2}} \ldots x_{k}^{\ell_{k}} R\left(x_{k+1}, \ldots, x_{n}\right)
$$

$$
+ \text { lower order terms in } x_{1}, x_{2}, \ldots, x_{k}
$$

where $R\left(x_{k+1}, \ldots, x_{n}\right)$ has character $H_{n-k, k}\left(\ell_{k+1}, \ldots, \ell_{n}\right)$. 
EXAMPLE 3.

$$
P_{3,1}(\mathrm{x})=\left(x_{1}-x_{2}\right)^{2}\left(x_{1}-x_{3}\right)^{2}\left(x_{2}-x_{3}\right)^{2}
$$

is Example 1 in the case $n=3$. Here we have $\mathcal{M}\left(P_{3,1}\right)=x_{1}^{4} x_{2}^{2} x_{3}^{0}$ and $\ell_{1}=4, \ell_{2}=2$, $\ell_{3}=0$. We may also express

$$
\begin{aligned}
P_{3,1}(\mathrm{x}) & =\sum_{m_{1}=0}^{4} \sum_{m_{2}=0}^{4} x_{1}^{m_{1}} x_{2}^{m_{2}} P_{m_{1} m_{2}}\left(x_{3}\right) \\
& =x_{1}^{4} x_{2}^{2}-2 x_{1}^{3} x_{2}^{3}+x_{1}^{2} x_{2}^{4}+\text { terms of lower total degree in } x_{1}, x_{2} .
\end{aligned}
$$

We next extablish several further properties of an arbitrary polynomial $P(\mathbf{x})$ satisfying conditions (a), (b), (c) above.

LEMMA 2. Suppose that $P(\mathrm{x})$ has character $H_{n, k}\left(\ell_{1}, \ldots, \ell_{n}\right)$, and that $n \geqslant$ $k+1$. Then $\ell_{1} \geqslant \ell_{k+1}+2$.

Proof: As in the previous example, express $P(\mathbf{x})$ as

$$
P(\mathrm{x})=\sum_{\text {each } m_{i} \geqslant 0} P_{m_{1}, m_{2}, \ldots, m_{k+1}}\left(x_{k+2}, \ldots, x_{n}\right) x_{1}^{m_{1}} x_{2}^{m_{2}} \ldots x_{k+1}^{m_{k+1}}
$$

thereby defining the coefficients $P_{m_{1}, \ldots, m_{k+1}}\left(x_{k+2}, \ldots, x_{n}\right)$.

Since $P(\mathbf{x})$ is totally symmetric, each $P_{m_{1}, \ldots, m_{k+1}}\left(x_{k+2}, \ldots, x_{n}\right)$ depends only on the values $\left\{m_{1}, m_{2}, \ldots, m_{k+1}\right\}$ and not on the order of these integers. From the hierarchical principle, the leading term of $P\left(x_{1}, x_{2}, \ldots, x_{n}\right)$ is

$$
x_{1}^{\ell_{1}} x_{2}^{\ell_{2}} \ldots x_{k+1}^{\ell_{k+1}} P_{\ell_{1}, \ldots, \ell_{k+1}}\left(x_{k+2}, \ldots, x_{n}\right) \text {. }
$$

The characteristic property (c) above implies that

$$
P\left(x, x, \ldots, x, x_{k+2}, \ldots, x_{n}\right) \equiv 0
$$

and hence its leading coefficient in the variable $x$ is zero. It is essential that this leading term has at least two source terms of the type displayed in (13). Otherwise, the characteristic property (c) above would imply that the leading term would vanish, and so would $P(\mathrm{x})$. We use this argument below.

Now suppose that the lemma is false. Then either

(i) $\ell_{1}=\ell_{2}=\ldots=\ell_{k+1}=\ell$ (say), or

(ii) $\ell_{1}=\ell_{2}=\ldots=\ell_{s}=\ell$ (say), and

$$
\ell_{s+1}=\ell_{s+2}=\ldots=\ell_{k+1}=\ell-1 \text { for some } s \in[1, k] .
$$


CASE (I). There is precisely one source term

$$
P_{\ell, \ell, \ldots, \ell}\left(x_{k+2}, \ldots, x_{n}\right) x_{1}^{\ell} x_{2}^{\ell} \ldots x_{k+1}^{\ell}
$$

in (14) contributing to the leading monomial $x^{(k+1) l}$. The characteristic property (c) above implies that this term is zero, contradicting the hypothesis that it is a leading term. Therefore Case (i) cannot hold.

CASE (II). There are now several source terms in (14) contributing to the coefficient of $x^{\ell_{1}+\ell_{2}+\ldots+\ell_{k+1}}$ in $P\left(x, \ldots, x, x_{k+2}, \ldots, x_{n}\right)$, which is clearly the leading monomial in $x$, and in fact they all provide equal contributions. The leading monomial of $P(x)$ is

$$
t(\mathbf{x}):=x_{1}^{\ell} x_{2}^{\ell} \ldots x_{s}^{\ell} x_{s+1}^{\ell-1} \ldots x_{k+1}^{\ell-1} \underbrace{\ell, \ell, \ldots, \ell}_{s \text { indices }}, \underbrace{\ell-1, \ldots, \ell-1}_{k+1-s \text { indices }}\left(x_{k+2}, \ldots, x_{n}\right)
$$

By permuting $x_{1}, \ldots, x_{k+1}$ in $t(x)$, and because the $P$ coefficient in the right-hand side of (15) is symmetrical with respect to the order of its subscripts, we only find terms which make a contribution to $P\left(x, x, \ldots, x, x_{k+2}, \ldots, x_{n}\right)$ of $x^{\ell_{1}+\ell_{2}+\ldots+\ell_{k+1}}$ $P_{\ell, \ldots, \ell, \ell-1, \ldots, \ell-1}\left(x_{k+2}, \ldots, x_{n}\right)$.

The characteristic property (c) above implies that the total contribution is zero, contradicting the hypothesis that $t(x)$ in $(15)$ is a leading term and so is non-zero. Therefore Case (ii) cannot hold.

Lemma 3. Suppose that $P(\mathbf{x})$ has character $H_{n, k}\left(\ell_{1}, \ldots, \ell_{n}\right)$. Then

$$
\ell_{1} \geqslant 2\left[\frac{n-1}{k}\right]
$$

and

$$
\operatorname{deg}\{P(x)\} \geqslant \lambda_{n, k}
$$

ProOF: By induction on $n$. The result is true for $n \leqslant k$ because $\lambda_{n, k}=0$ for $0 \leqslant n \leqslant k$. Suppose now that $n \geqslant k+1$, and that the lemma holds for $n$ replaced by any smaller number.

From the representation (12), and the induction hypothesis with $n$ replaced by $n-k$,

$$
\ell_{k+1} \geqslant 2\left[\frac{n-k-1}{k}\right]
$$

From Lemma 2,

$$
\ell_{1} \geqslant 2+\ell_{k+1} \geqslant 2\left[\frac{n-1}{k}\right]
$$


and (16) is proved. For (17), we use the representation (11) and the induction hypothesis with $n$ replaced by $n-1$. Then

$$
\operatorname{deg}\{P(\mathbf{x})\} \geqslant \ell_{1}+\lambda_{n-1, k} \geqslant \lambda_{n, k} \text {. }
$$

LEMMA 4. Suppose that $\boldsymbol{P ( x )}$ is a polynomial which has character $H_{n, k}\left(\ell_{1}, \ell_{2}, \ldots, \ell_{n}\right)$ and with minimal total degree

$$
d_{n, k}=\ell_{1}+\ell_{2}+\ldots+\ell_{n}
$$

Then

$$
d_{n, k}=\lambda_{n, k},
$$

and

$$
\ell_{1}=2\left[\frac{n-1}{k}\right]
$$

Proof: Result (18) follows from the fact that (10) shows that equality is possible in (17), using the generalised discriminant. From (11), (17) and (18), we have $\ell_{1}=d_{n, k}-\operatorname{deg}\{Q\} \leqslant \lambda_{n, k}-\lambda_{n-1, k}$. But from (16),

$$
\ell_{1} \geqslant 2\left[\frac{n-1}{k}\right]=\lambda_{n, k}-\lambda_{n-1, k}
$$

and therefore

$$
\ell_{1}=\lambda_{n, k}-\lambda_{n-1, k}=2\left[\frac{n-1}{k}\right] .
$$

These preliminaries are the principal requirements for our main theorem:

TheOREM 1. The generalised discriminant theorem. The non-trivial symmetric polynomial $P\left(x_{1}, x_{2}, \ldots, x_{n}\right)$ of least total degree (with coefficients in a field of characteristic zero) which vanishes identically when any $k+1$ of its variables $x_{i}$ are equal is unique up to an overall scale factor, and it is called the generalised discriminant $P_{n, k}(\mathrm{x})$. It may be expressed as

$$
P_{n, k}(\mathbf{x})=\sum_{\sigma \in S_{n}} \sigma\left\{\prod_{\substack{\ell \\ \ell=1}} \prod_{\substack{i<j \\ i, j \in I_{l}}}\left(x_{i}-x_{j}\right)^{2}\right\}
$$

where $\sigma$ denotes any permutation of the indices $1,2, \ldots, n$. These indices have been grouped into disjoint sets $I_{1}, I_{2}, \ldots, I_{k}$. The sets $I_{1}$, then $I_{2}$, then $I_{3}, \ldots$, 
then $I_{r}$ each consist of $q+1$ indices taken sequentially from $1,2, \ldots, r(q+1)$ and $I_{r+1}, I_{r+2}, \ldots, I_{k}$ consist of $q$ indices similarly taken from $r(q+1)+1, r(q+1)+$ $2, \ldots, n$. Here $q=[n / k]$ and $r=n \bmod k$.

The minimal degree of $P(\mathbf{x})$ is

$$
\operatorname{deg}\left\{P_{n, k}(\mathbf{x})\right\}=k q(q-1)+2 r q
$$

ProOf: The representation (20) is the same as (4), except that the indices are used in natural order for descriptive simplicity. The result (21) follows from (5) and (18), and the observation that (21) is trivial for $n \leqslant k$, because both $P(x)$ and $P_{n, k}(x)$ are nonzero constants (they contain too few variables to make equal).

The rest of the proof is by induction on $n$. From (11), (19) and the induction hypothesis,

$$
\begin{gathered}
P(\mathbf{x})=\alpha x_{1}^{2[(n-1) / k]} P_{n-1, k}\left(x_{2}, x_{3}, \ldots, x_{n}\right) \\
+ \text { lower order terms in } x_{1},
\end{gathered}
$$

for some non-zero constant $\alpha$. From (4), (8) and (9),

$$
\begin{gathered}
P_{n, k}(\mathbf{x})=\beta x_{1}^{2[(n-1) / k]} P_{n-1, k}\left(x_{2}, x_{3}, \ldots, x_{n}\right), \\
+ \text { lower order terms in } x_{1},
\end{gathered}
$$

for some non-zero constant $\beta$. Therefore $S(\mathbf{x}):=\alpha P_{n, k}(\mathbf{x})-\beta P(\mathbf{x})$ is a polynomial of total degree $\lambda_{n, k}$ and some character $H_{n, k}\left(\ell_{1}^{\prime}, \ell_{2}^{\prime}, \ldots, \ell_{n}^{\prime}\right)$ but degree less than $2[(n-1) / k]$ in $x_{1}$. This would contradict Lemma 4 , unless $S(\mathrm{x}) \equiv 0$.

\section{Conclusion}

The generalised discriminant $P_{n, k}(\mathrm{x})$ does not appear to have been considered previously despite its fairly natural appearance. In the case $k=1$, any symmetric polynomial $T(x)$ in the variables $x_{1}, x_{2}, \ldots, x_{n}$ which vanishes when any two of these variables are equal must be divisible by the ordinary discriminant $P_{n, 1}(x)$ [4]. The corresponding result for the generalised discriminant $P_{n, k}(\mathbf{x})$ is not true when $k>1$, and this fact presumably accounts for its lower profile. A similar phenomenon arises for certain 'generalised resultants' which were constructed by Pragacz [5]; these turn out to be super Schur functions [3].

Generalised discriminants were originally introduced to solve the problem of how the formula for the denominator polynomial of a vector-valued Padé approximant reduces in the scalar case [1]; the solution was given by Woodcock and Graves-Morris [7] but assuming the results of this paper. 


\section{References}

[1] P.R. Graves-Morris, 'Problem 92-20', SIAM Review 34 (1992), 649-650.

[2] S. Lang, Algebra, Chapter 5 Section 9 (Addison-Wesley, Reading, Mass., 1967).

[3] I.G. Macdonald, Symmetric functions and Hall polynomials (Oxford University Press, Oxford, 1979).

[4] M. Marcus, Introduction to modern algebra (Dekker, New York, 1978).

[5] P. Pragacz, 'A note on the elimination theory', Nederl. Akad. Wetensch. Indag. Math. 49 (1987), 215-221.

[6] R.J. Wilson, Introduction to graph theory (Longman, London, 1985).

[7] C.F. Woodcock and P.R. Graves-Morris, 'Solution to Problem 92-20', SIAM Review 35 (1993), 649-651.

Institute of Mathematics and Statistics

University of Kent

Canterbury

Kent CT2 7NF

England
Department of Mathematics and Statistics University of Canterbury

Private Bag 4800

Christchurch

New Zealand 